\title{
Expanding the Phenotype of the X-linked BCOR Microphthalmia syndromes
}

Nicola Ragge ${ }^{1,2}$, Bertrand Isidor ${ }^{3}$, Pierre Bitoun ${ }^{4}$, Sylvie Odent 5 , Irina Giurgea 6, Benjamin Cogné ${ }^{3}$, Deb Wallid ${ }^{3}$, Marie Vincent ${ }^{3}$, Jessica Le Gall ${ }^{3}$, Jenny Morton ${ }^{2}$, Derek Lim ${ }^{2}$, DDD study ${ }^{7}$, Guylène Le Meur ${ }^{8}$, Celia Zazo Seco ${ }^{9}$, Dimitra Zafeiropoulou 10, Dorine Bax ${ }^{1}$, Petra Zwijnenburg 11, Anara Arteche ${ }^{12}$, Saoud

Tahsin Swafiri 12, Ruth Cleaver 13, Meriel McEntagart 13, Usha Kini 14, William Newman 15, Carmen Ayuso 12,16, Marta Corton 12,16, Yvan Herenger 17, Médéric Jeanne 17, Patrick Calvas 9,18, Nicolas Chassaing 9,18

1 Department of Biological and Medical Sciences, Faculty of Health and Life Sciences, Oxford Brookes University, Oxford, UK

2 West Midlands Regional Clinical Genetics Service and Birmingham Health Partners, Birmingham Women's and Children's Hospital NHS Foundation Trust, Birmingham, UK

3 Service de génétique médicale, Hôtel-Dieu, CHU de Nantes, France 4 SIDVA 91, Ophthalmic Genetics, 1 rue de la Cour de France, 91260 Juvisy s/orge, France

5 Service de Génétique Clinique, Centre de référence CLAD-Ouest, Université Rennes 1, UMR 6290 CNRS IGDR, CHU Rennes, Rennes, France 6 U.F. de Génétique moléculaire, Hôpital Armand Trousseau, Assistance Publique - Hôpitaux de Paris; INSERM UMR S933, Faculté de médecine, Sorbonne Université, Paris 75012, France

7 DDD Study, Wellcome Trust Sanger Institute, Hinxton, Cambridge, UK 8 Service d'ophtalmologie, Hôtel Dieu, CHU de Nantes, France 9 UDEAR, UMR 1056 Inserm - Université de Toulouse, Toulouse, France 10 Department of Human Genetics, Radboud University Medical Centre, Geert Grooteplein 10, 6525 GA Nijmegen, The Netherlands

11 Dept of Clinical Genetics, VU University Medical Center, Amsterdam, the Netherlands

12 Department of Genetics, Health Research Institute-Jiménez Díaz Foundation, University Hospital (IIS-FJD-UAM) Madrid, Spain. 
13 South West Thames Regional Genetics Service, St. George's Healthcare NHS

Trust, London, UK

14 Oxford Centre for Genomic Medicine, Oxford, UK

15 Manchester Royal Eye Hospital, Manchester, UK

16 Centre for Biomedical Network Research on Rare Diseases (CIBERER), ISCIII, Madrid, Spain

17 Service de Génétique Médicale, CHU de Tours, Tours, France

18 Department of Medical Genetics, CHU Toulouse, Purpan Hospital, 31059

Toulouse, France

Corresponding Author:

Nicola Ragge MD FRCPCH FRCP FRCOphth

Email: nragge@brookes.ac.uk; Nicola.ragge@bwnft.nhs.uk;

Telephone: +44 1865 484413; +441213358024

orcid.org/0000-0002-6122-4521

Key words: BCOR gene, anophthalmia, microphthalmia, X-linked, Oculofaciocardiodental (OFCD) syndrome, lymphoma 


\section{Acknowledgements}

We acknowledge generous support from the families published in this article. This work was supported by grants from the Clinical Research Hospital Program from the French Ministry of Health (PHRC 09109 01), the Fondation of France, the Fondation Maladies Rares, Berthe Fouassier, Rétina France, VICTA (Visually Impaired Children Taking Action), MACS (Microphthalmia, Anophthalmia Coloboma Support), Baillie Gifford, Spanish Institute of Health Carlos III (CP12/03256), Spanish Ministry of Economy and Competitiveness (SAF201346943-R) and Mutua Madrileña Foundation. The DDD study presents independent research commissioned by the Health Innovation Challenge Fund [grant number HICF-1009-003], a parallel funding partnership between the Wellcome Trust and the Department of Health, and the Wellcome Trust Sanger Institute [grant number WT098051]. The views expressed in this publication are those of the authors and not necessarily those of the Wellcome Trust or the Department of Health. The study has UK Research Ethics Committee approval (10/H0305/83, granted by the Cambridge South REC, and GEN/284/12 granted by the Republic of Ireland REC). The research team acknowledges the support of the National Institute for Health Research, through the Comprehensive Clinical Research Network. This study makes use of DECIPHER (http://decipher.sanger.ac.uk), which is funded by the Wellcome Trust. 


\section{Abstract}

4 Two distinct syndromes arise from pathogenic variants in the X-linked gene BCOR (BCL-6 corepressor): OculoFacioCardioDental (OFCD) syndrome, which

6 affects females, and a severe microphthalmia ('Lenz'-type) syndrome affecting males. OFCD is an X-linked dominant syndrome caused by a variety of BCOR null p.(Pro85Leu). Here we detail 16 new cases (11 females with 4 additional,

13 genetically confirmed, affected female relatives; 5 male cases each with

14 unaffected carrier mothers). We describe new variants and broaden the

15 phenotypic description for OFCD to include neuropathy, muscle hypotonia,

16 pituitary underdevelopment, brain atrophy, lipoma and the first description of

17 childhood lymphoma in an OFCD case. Our male X-linked recessive cases show

18 significant new phenotypes: developmental delay (without eye anomalies) in 2

19 affected half-brothers with a novel BCOR variant, and one male with high myopia,

20 megalophthalmos, posterior embryotoxon, developmental delay, and heart and

21 bony anomalies with a previously undescribed BCOR splice-site variant. Our

22 female OFCD cases and their affected female relatives showed variable features,

23 but consistently had early onset cataracts. We show that a mosaic carrier

24 mother manifested early cataract and dental anomalies. All female carriers of

25 the male X-linked recessive cases for whom genetic confirmation was available

26 showed skewed X-inactivation and were unaffected. In view of the extended

27 phenotype, we suggest a new term of X-linked BCOR-related syndrome. 
30 Oculofaciocardiodental (OFCD) and severe X-linked microphthalmia syndromes

31 are related conditions caused by allelic pathogenic alterations in BCOR (BCL-6

32 corepressor). OFCD is an X-linked dominant condition, affecting females

33 (presumed male lethal), with examples of mother-to-daughter transmission.

34 Skewed X-inactivation (90-100\%) has been demonstrated in informative cases

35 (Ng, Thakker et al. 2004). It is characterised by the pathognomonic association of

36 congenital or early onset cataract with dental anomalies (including

37 radiculomegaly, delayed primary/secondary dentition, hypodontia, fusion of

38 teeth), with a variety of other features. These other features are principally

39 ocular (microphthalmia, cataract, glaucoma, retinal detachment), cardiac (septal

40 defects), skeletal (hammer toes or camptodactyly, 2-3 toe syndactyly, broad

41 halluces, radioulnar synostosis, scoliosis), and facial anomalies (cleft palate,

42 septate nasal cartilage, long narrow face, arched eyebrows). Less freqently they

43 include mild developmental delay (11\%), posterior fossa anomalies (in a fetal

44 loss), hearing impairment (9\%) and defects of laterality (situs inversus, asplenia)

45 in a single case (Ng, Thakker et al. 2004, Horn, Chyrek et al. 2005, Oberoi, Winder

46 et al. 2005, Hilton, Johnston et al. 2009, Davoody, Chen et al. 2012, Lozic,

47 Ljubkovic et al. 2012, Kantaputra 2014, Surapornsawasd, Ogawa et al. 2015, Ma,

48 Grigg et al. 2016). In typical OFCD cases, BCOR is affected by a variety of null

49 variants: nonsense, splicing, frameshift, deletions of part or all of the coding

50 sequence, predicted to lead to nonsense mediated decay. Asymptomatic mosaic

51 female carriers have been described: Hilton and colleagues refer to the

52 asymptomatic mother of case XVII; mosaicism was estimated by a reduction of

53 the Sanger sequencing peak to $75 \%$ as opposed to $50 \%$ for her fully manifesting

54 daughter (Hilton, Johnston et al. 2009). Furthermore, individuals with BCOR

55 pathogenic variants with mainly ocular features are also reported ( $\mathrm{Ng}$, Thakker

56 et al. 2004; Hilton, Johnston et al. 2009; Ma, Grigg et al. 2016).

58 BCOR pathogenic variants have also been identified in affected males with X-

59 linked recessive severe ('Lenz') microphthalmia. In 2004, the missense variant

60 c.254C $>$ T, p.(Pro85Leu)_was identified in an affected male, and segregated with

61 disease phenotype ( $\mathrm{Ng}$, Thakker et al. 2004) and since then further cases 
62 described (Hilton, Johnston et al. 2009, Suzumori, Kaname et al. 2013).

63 Recently, a de novo novel BCOR missense variant (c.1619G>A; pArg540Gln) was

64 identified in a boy with congenital glaucoma, complex cardiac anomalies,

65 dextrocardia and cerebral white matter hypoplasia, following sequencing

66 analysis of PITX2, FOXC1 and BCOR (Zhu, Dai et al. 2015). Although the causative

67 nature of this missense variant could not be established with certainty,

68 supporting evidence from in silico analysis and absence of variant from control

69 cohorts was highly suggestive.

71 Only a small percentage of males with severe microphthalmia $(<1 \%$ in our series, unpublished data), even with an X-linked inheritance pattern, carry BCOR variants (Hilton, Johnston et al. 2009). The features described in males harboring the p.(Pro85Leu) variant in BCOR include: bilateral microphthalmia or anophthalmia, microcephaly, hypoplastic corpus callosum, mild-severe developmental delay, radioulnar synostosis, simple ears, no dental anomalies, cardiac anomalies, multiple partial finger syndactyly, fifth finger clinodactyly, and hypospadias (Ng, Thakker et al. 2004, Hilton, Johnston et al. 2009, Suzumori, Kaname et al. 2013). Although the features of so-called 'Lenz' microphthalmia are broader (Lenz 1955, Traboulsi, Lenz et al. 1988), some of these may be explained by the newly described genes HMGB3 (Scott, Mohr et al. 2014) and NAA10 which have been identified as other causes of X-linked 'Lenz' microphthalmia syndrome (Esmailpour, Riazifar et al. 2014).

Here, using whole exome and targeted gene sequencing, we identified 16 further index cases (15 families) with pathogenic variants in BCOR. The female cases comprised of eleven females with OFCD, and additionally 3 affected mothers and 1 affected sister manifesting variable phenotypes. The five male index cases comprised of two unrelated cases with the recurrent c.254C $>\mathrm{T}$ (p.Pro85Leu) variant and manifesting a severe microphthalmia syndrome, two half-brothers with a previously undescribed c.4807A>C (p.Ser1603Arg) variant with developmental delay and posterior embryotoxon, and one boy with high myopia, posterior embryotoxon, severe developmental delay, wrist and finger anomalies with a previously undescribed splice site variant c.4741+1G>A (p.[?]). We 
95 review the literature and show that male cases have demonstrably high

96 prevalence of cardiac, skeletal, craniofacial, and genitourinary anomalies in

97 addition to their well described severe eye anomalies and developmental delay.

98 We also show a surprising proportion of female OFCD cases with skeletal

99 anomalies, hearing loss and developmental delay, and one with childhood

100 lymphoma.

101

102 Materials and Methods

103 Patient cohort

104 Cases 1-9 were recruited as part of a UK national study of developmental eye

105 anomalies and a French cohort of microphthalmic or anophthalmic patients.

106 Informed consent was obtained from all individuals in the study in accordance

107 with Ethics Approval obtained for the study from Cambridgeshire 1 Ethics

108 Committee 04/Q0104/129 (UK patients) and local Ethics Committee (CPP Sud-

109 Ouest et Outre-Mer II) (French patients). Case 13 was recruited as part of a

110 Spanish study of congenital ocular anomalies approved by the Ethics Committee

111 of the Fundación Jiménez Díaz University Hospital. Cases 10 and 14 were

112 recruited to the DDD (Deciphering Developmental Disorders) Study, which has

113 UK Research Ethics Committee approval (10/H0305/83, granted by the

114 Cambridge South REC, and GEN/284/12 granted by the Republic of Ireland REC).

115 Cases 11, 12, 15 and 16 were consented for diagnostic genetic testing: single

116 gene, or whole exome sequencing (WES). Additional informed consent was

117 obtained from all individual participants for whom identifying information is

118 included in this article.

120 Methods

121 Whole exome sequencing (cases $1,4,5,9,10,12,13,14$ )

122 WES was undertaken in 24 previously undiagnosed UK/French eye anomaly

123 patients (12 males and 12 females). Case 4 had exome sequencing because of the

124 association of developmental delay, dysmorphic features and posterior

125 embryotoxon. Exome capture was performed using the Nimblegen V3

126 Enrichment kit following the manufacturer's protocol. The captured libraries

127 were sequenced with an Illumina HiSeq2000 with 100-bp paired-end reads. We 
used PolyPhen-2, SIFT and Mutation Taster software tools to predict the

129 functional effects of variants (Adzhubei, Schmidt, et al, 2010; Ng, Hennekoff et al,

130 2003; Schwarz, Cooper, et al, 2014). This strategy allowed the identification of

131 BCOR variants in cases 1,5 and 9. Case 12 had similar WES, but not captured and

132 instead using an Illumina HiSeq2500 and 125-bp paired end sequencing. For

133 cases 10 and 14, identified via the DDD study, trio-based exome sequencing was

134 performed on the affected individual and their parents, as previously described

135 (Wright, Fitzgerald et al. 2015). Case 13 had targeted clinical exome sequencing.

136 Libraries were prepared using TruSightOne (Illumina) following the

137 manufacturer's protocol. The captured libraries were sequenced with an

138 Illumina NextSeq500 with 150-bp paired-end reads.

140 Targeted resequencing of 187 genes (case 2)

141 Targeted resequencing of 187 genes, including BCOR, was performed for 96

142 UK/French individuals (44 males and 52 females) with undiagnosed

143 microphthalmia or anophthalmia. 600ng of subject genomic DNA was used to

144 capture the 2310 coding exons using a custom Agilent SureSelect Target

145 Enrichment System kit. Sequence capture, enrichment and elution were

146 performed according to manufacturer's instruction and protocols (SureSelect,

147 Agilent) without modification except for library preparation performed with the

148 NEBNext $\AA$ Ultra kit (New England Biolabs $\AA$ ). Libraries were pooled and

149 sequenced on an Illumina HiSeq2000 as paired-end 75bp reads. We used

150 PolyPhen-2, and SIFT software tools to predict the functional effects of variants. This

151 strategy allowed the identification of the BCOR variant in case 2.

153 Sanger sequencing

154 All pathogenic $B C O R$ variants retained after filtering from the whole exome or

155 targeted resequencing data were confirmed by Sanger sequencing. Parental

156 studies were performed to determine whether these variants were inherited or

157 appeared de novo. Direct BCOR (NM_001123385.1, 1755 aa) sequencing was

158 performed in the other female cases $(3,6,7,8,11,13,14,15)$ and one male case

159 (2) because of suggestive features and to confirm WES findings.

160 Case 16 had a copy number variant detected by array Comparative Genomic 
161 Hybridisation (CGH) (Agilent 60k ISCA) and confirmed by qPCR.

162

163 Non-random X-inactivation analysis

164 This was performed using a fluorescent PCR assay encompassing the X

165 chromosome androgen receptor CAG repeat polymorphism. The methylation

166 sensitive restriction enzyme, HpaI was used for prior digestion of genomic DNA.

167 X-inactivation classification ratios: Complete skewing=100:1; Highly

168 skewed=90:10-99:1; Moderate skewing=80:20-89:11; Random X-

169 inactivation=50:50-79:21 (Amos-Landgraf, Cottle et al, 2006).

171 Assessment of Mosaicism

172 This was performed using semi-quantitative multiplex fluorescent PCR (QMF-

173 PCR) analysis of the BCOR gene to determine the level of mosaicism. This method

174 was previously published as described in the paper by Hilton and colleagues

175 (Hilton, Johnston, et al, 2009).

176

177 Literature review

178 Published cases with pathogenic BCOR variants were identified through previous

179 literature reviews and Pubmed searches. Variants were annotated using a

180 common reference sequence, NM_001123385.1, and all variants were checked

181 using mutalyzer (www.mutalyzer.nl)(Wildeman, van Ophuizen et al. 2008).

182

183 Case Descriptions (Table 1)

184 Case 1

185 Case 1 is a 13-year-old Caucasian girl with right microphthalmia with dense

186 cataract and left microphthalmia with secondary aphakia, secondary glaucoma

187 and a left visual acuity of 20/300. She was born at full term following ultrasound

188 scans during pregnancy that detected choroid plexus cysts. Early cranial

189 Magnetic Resonance Imaging (MRI) revealed a corpus callosal lipoma. She had

190 early onset cataracts and left cataract surgery at 6 weeks of age. Her

191 development was slightly delayed: she smiled at 3-4 months, sat at 10 months

192 and walked at 23 months of age. Her speech was normal. She had recurrent

193 urinary tract infections (normal renal ultrasound), and growth hormone 
deficiency diagnosed at 3 years of age and treated with growth hormone. She had late eruption of her first teeth and delayed loss of first teeth at 9 years of age. She had unusual positioning of her adult teeth, which were small, with a second row of teeth.

At 11 years of age her growth had reached: height $2^{\text {nd }}$ centile, weight $<0.4^{\text {th }}$ centile and head circumference $50^{\text {th }}$ centile. She had long slender fingers and hyperextensible joints. Her feet showed an increased sandal gap and she had right second toe clinodactyly (Figure 1a-e). She had reduced bladder control and decreased reflexes. WES revealed a de novo heterozygous variant in $B C O R$ c.2428C >T p.(Arg810*). This variant has been previously described as causing OFCD with a strikingly similar phenotype in a paper by Hilton and colleagues (case X) (Hilton, Johnston et al. 2009) (see Supplementary Table 1).

\section{Case 2}

This 21-year-old Caucasian male first presented to the ophthalmic genetics clinic at the age of 9 years with bilateral microphthalmia (Figure 1f-i). He was born at term, birth weight unknown. He had delayed motor milestones and walked with assistance by 4 years of age. He had bilateral cryptorchidism and vesico-ureteric reflux, requiring surgical correction at 7 years of age after repeated pyelonephritis. His social development was delayed; he was diagnosed with autistic spectrum disorder. There was a family history of paternal bilateral cataracts requiring surgery at the age of 30 years, and low vision. His mother had stellate irides and he had a healthy younger sister.

At 9 years-of-age, he had no speech and difficulty swallowing, tolerating only liquid food. He suffered from primary enuresis and had undergone surgical correction for severe scoliosis. He suffered from recurrent dental infections requiring dental extraction. His height was $1.25 \mathrm{~m}$ (9th centile), weight $20.5 \mathrm{~kg}$ (0.4-2 nd $c e n t i l e)$ and head circumference $53 \mathrm{~cm}$ (25 th centile). He had bilateral severe microphthalmia with no light perception, short (10 $\mathrm{mm})$ downslanting palpebral fissures and secondary midface hypoplasia. He had a long face with abundant hair, tall forehead, thick eyebrows, a broad nasal root and tip, a long philtrum, thin upper lip and thick lower lip. He had large low set posteriorly 
227 rotated ears with prominent antihelices. He had a thin body habitus with a

228 barrel-shaped chest, long slender fingers with fifth finger clinodactyly, and broad

229 halluces. He showed hypotonia with reduced muscle mass and marked

230 ligamentous laxity. His cranial MRI was normal.

232 At the age of 21 years, his height was $1.6 \mathrm{~m}\left(0.4-2^{\text {nd }}\right.$ centile $)$, weight $40.5 \mathrm{~kg}\left(<3^{\text {rd }}\right.$

233 centile) and head circumference $55 \mathrm{~cm}$ ( $90^{\text {th }}$ centile). He had limited speech of a

234 few words. He walked with a spastic gait, had poor muscle mass and suffered

235 from scoliosis. He attended a daytime assisted care facility, functioning

236 reasonably independently, and played the piano.

238 Genetic testing of SOX2, OTX2, VSX2, RAX, and FOXE3 were normal. Targeted

239 sequencing identified a BCOR c.254C >T; p.(Pro85Leu) variant inherited from his

240 healthy mother. His maternal aunt had previously had a termination of

241 pregnancy for a severely malformed male fetus suspected of trisomy 13, without

242 genetic analysis or fetal pathology examination. She may be a carrier of the same

$243 B C O R$ variant, although genetic analysis was declined.

\section{Case 3}

246 This 3-year-old Caucasian girl was born with bilateral microphthalmia and

247 cataract (Figure 1j) having had bilateral congenital cataract detected at 22 weeks

248 of pregnancy. She also had an atrial septal defect (ASD) that was managed

249 conservatively. The cataracts were removed without intraocular lens

250 implantation at 2 months of age, with a subsequent right vitrectomy for capsular

251 opacity at 2 years of age. However, she developed a T-cell lymphoma (stage III

252 on St Jude's classification) at the age of 12 months and was treated with

253 chemotherapy, achieving remission after 2 years of treatment. She had late

254 eruption of her first teeth and abnormal crown volume on the upper maxillary

255 canines and central incisors on the palatal side, without any misalignment of the

256 teeth. All primary and permanent teeth were present on the head Computerised

257 Tomography (CT) scan. At the age of 3 years her microphthalmic eyes measured

258 right eye (RE) corneal diameter of $9.5 \mathrm{~mm}$, axial length of $17.59 \mathrm{~mm}$ and left eye 
(LE) corneal diameter of $8.5 \mathrm{~mm}$, axial length of $16.06 \mathrm{~mm}$, with an increase of corneal thickness RE $635 \mu \mathrm{m}$ and LE $680 \mu \mathrm{m}$. At 4 years, following patching, she achieved visual acuities of RE 0.7 logMar and LE 0.2 logMar.

263 She had normal growth and no developmental delay. She had long slender

264 fingers and hands, downslanting, dysplastic ears and a high arched narrow

265 palate. Targeted analysis of BCOR revealed the de novo variant

266 c.1209_1210delCC; p.(Gln404Ala $\left.f^{*} 35\right)$, predicted as pathogenic.

Case 4

269 Case 4 is an 18-month-old Caucasian boy, only child of unrelated healthy parents.

270 He has 3 half-brothers on his mother's side, including case 5 (Figure 2A). He was

271 born at 38 weeks' gestation with a birth weight $2.875 \mathrm{~kg}$ ( $25^{\text {th }}$ centile), length

$27250.7 \mathrm{~cm}$ (80 $80^{\text {th }}$ centile) and head circumference $33.5 \mathrm{~cm}\left(28^{\text {th }}\right.$ centile $)$. He had a

273 large ASD. Ophthalmological examination showed bilateral posterior

274 embryotoxon. He had distinctive features, including large earlobes, long fingers

275 with $4^{\text {th }}$ and $5^{\text {th }}$ camptodactyly, and short and deep-set toenails and a left

276 temporal haemangioma. His growth was normal, however, he had some

277 developmental delay: he sat at 12 months, at 18 months of age he could not stand

278 unsupported; he could say one word. While his array CGH was normal, WES

279 revealed the variant c.4807A>C; p.(Ser1603Arg) in BCOR, inherited from his

280 asymptomatic mother. This variant involves a highly conserved amino acid, is

281 absent from the general population (gnomAD database) (Lek, Karczewski, et al,

282 2016) and is predicted to be deleterious by in silico software Polyphen-2, SIFT

283 and Mutation Taster.

\section{Case 5}

286 Case 5 is a 5-year-old Caucasian boy, half-brother of case 4. He was born at full 287 term with asymmetrical intrauterine growth retardation. His birth weight was $2882.860 \mathrm{~kg}$ ( $5^{\text {th }}$ centile), length $50 \mathrm{~cm}$ ( $37^{\text {th }}$ centile) and OFC $33.5 \mathrm{~cm}$ (12 th centile). 289 He had initial feeding difficulties and presented with posterior cleft palate, major 290 axial hypotonia with highly hypertonic limbs, and a large ASD. Ophthalmological 291 examination at birth showed bilateral posterior embryotoxon. He also had 
asymmetrical dysplastic ears, camptodactyly of all fingers, fetal toe pads, and multiple capillary malformations. He had severe developmental delay; he spoke

294 fewer than 10 words at 5 years and walked at 4 years and 10 months. He had 295 severe feeding difficulties causing initial growth retardation, but at age $4 \frac{1}{2}$ years 296 his weight was $17.5 \mathrm{~kg}$ (0 SD), height $100.5 \mathrm{~cm}(-1 \mathrm{SD})$ and OFC $48.5 \mathrm{~cm} \mathrm{(-2.5}$ 297 SD). His brain MRI showed a posterior arachnoid cyst. Sanger sequencing 298 revealed the same variant c.4807A>C; p.(Ser1603Arg) in BCOR as his brother 299 (case 4). This variant was absent in the two healthy brothers of cases 4 and 5.

Case 6

302 Case 6 is a 17-year-old Caucasian girl, first child of unrelated healthy parents, 303 with two unaffected siblings. She was born at 37 weeks' gestation and bilateral 304 congenital cataract was diagnosed at 1 month, with surgery performed at 3 305 months of age. Later she developed secondary bilateral glaucoma with significant 306 reduction in visual acuity, and received further surgery on the left eye at 7 years 307 of age. She developed a right retinal detachment at the age of 12 years and now 308 her visual acuity is RE 30/100 and LE no perception of light. She had delayed 309 replacement of her primary teeth with a secondary dentition. Radiographs 310 showed radiculomegaly; all teeth were present (Figure 10).

311 She had normal growth and development. She had distinctive facial features with 312 a short bulbous nose, microtia and prognathism. She also showed $5^{\text {th }}$ finger 313 clinodactyly and left 2-3 toe partial syndactyly.

314 Targeted BCOR analysis initiated by the geneticist at age 13 y 9 months revealed 315 the de novo variant c.4598_4616dup; p.(Glu1539Asp $f s^{* 7)}$, predicted as 316 pathogenic.

\section{Case 7}

319 Case 7 is a 15-year-old Caucasian girl, the second of three girls, born at full term 320 with bilateral microphthalmia and cataract (Figure 2B). She had bilateral 321 cataract surgery in the first months of life, but subsequently developed chronic 322 bilateral glaucoma with acute episodes, requiring surgery. Her visual acuity is 323 reduced to RE: light perception and LE: count fingers at $1 \mathrm{~m}$ wearing aphakia324 correcting glasses. She also had an ASD, corrected by cardiac surgery at the age 
325 of 4 years. She had delayed loss of her primary teeth, with radiculomegaly, 326 causing a misalignment of the teeth. All the primary teeth had to be removed in 327 order to enable permanent teeth to erupt. She had normal growth and

328 development. She exhibited distinctive facial features including broad nose, and 329 long, slender fingers and toes (Figure 1p-r).

330 Her mother had surgery in infancy for bilateral congenital cataract, had frequent 331 dental issues and suffered 8 miscarriages. Her maternal grandmother had at 332 least one miscarriage and bilateral early onset cataract. Her younger sister also 333 had surgery for bilateral congenital cataract and also had dental anomalies.

334 Targeted BCOR analysis initiated by the geneticist at 12 years of age revealed the 335 variant c.867G>A; p.(Trp289*), predicted as pathogenic. This variant was 336 inherited from the affected mother and was also present in the younger affected 337 sister.

\section{Case 8}

340 This 61/2-year-old girl was born at full term. At one month of age, after initial 341 feeding difficulties, she was diagnosed with bilateral congenital cataract and mild 342 microphthalmia. She had two large haemangiomas (one on the forehead, one in

343 the neck), a lipomatous lesion in the thyroid lobe diagnosed clinically and on

344 ultrasound and a thyroglossal cyst (Figure $1 \mathrm{~s}-\mathrm{w}$ ). At age $6 \frac{1}{2}$ years she had 345 normal growth and development. She had agenesis of both superior lateral 346 incisors and cutaneous syndactyly of second and third toes. Subsequent follow-

347 up revealed left ventricular noncompaction, without rhythm disturbance and 348 with good ventricular function, and a small persistent ductus arteriosus. Sanger 349 sequencing of BCOR revealed a frameshift variant c.2947_2948insTGCATACT; 350 p.(Glu983Valfs*41). The same variant was identified in her mother, who had 351 bilateral congenital cataract, microphthalmia and agenesis of the two lateral 352 incisors with large spacing of the two upper median incisors, but in a mosaic 353 state (about 20\% of mutated p.(Glu983Valfs*) alleles in blood).

356 This 27-year-old male was born at 38 weeks following a normal pregnancy 357 during which an ultrasound scan at 18/40 demonstrated urinary reflux and one 
kidney larger than the other. At birth he was diagnosed with bilateral anophthalmia, small palpebral apertures, hypotonia, moderate degree of chronic renal failure secondary to bilateral renal dysplasia with associated bilateral vesicoureteric reflux (corrected age 2 years) and urethral hypoplasia. He had normal growth and developmental milestones, and excellent musical and verbal skills. His mother had a history of neurofibromatosis type 1 and multiple strawberry nevi, but was otherwise healthy. His MRI scan was reported as normal. At age 27 years, he had normal growth parameters, with long fingers and toes, and large ears with squared off earlobes (Figure 1x-ac). WES revealed a maternally inherited BCOR c.254C>T; p.(Pro85Leu) variant.

Case 10

370 Case 10 is a 9 year-old-girl born at 38 weeks' gestation by Caesarian section due to delayed rupture of membranes. She had bilateral congenital cataract and microphthalmia with corneal diameters of $9 \mathrm{~mm}$, persistent fetal vasculature and small optic nerves. She was noted to have a prominent forehead, flat nasal bridge, upturned nose, mesaxial polysyndactyly (of $4^{\text {th }}$ digit) with $5 / 6$ syndactyly of the right hand and partial 2/3 syndactyly of the right toes (Figure 1ad-ai). She also had a moderate secundum ASD with a mildly dysplastic pulmonary valve on echocardiography. She had slight widening of her cerebral falx on cranial ultrasound. Her maternal grandmother had postaxial polydactyly on one hand and a maternal first cousin once removed had bilateral postaxial hand polydactyly. At 10 months of age, she had delayed motor milestones and was not yet sitting unsupported. She did not have any teeth yet and her anterior fontanelle was still open. She had fine hair, a short nose with slightly broad nasal tip, small mouth and narrow palate. She had surgery for her ASD at the age of 3 years. Interestingly, her growth parameters progressed from length $0.4^{\text {th }}$ centile, weight $25^{\text {th }}$ centile and head circumference $75^{\text {th }}$ centile at 1 month, to height and weight $9^{\text {th }}$ centile, and head circumference $75^{\text {th- }} 91^{\text {st }}$ centile at 10 months, and by 8 years-of-age she reached a height on the $91^{\text {st }}$ centile, weight $98-99.6^{\text {th }}$ centile and a head circumference of $58.9 \mathrm{~cm}$ ( $>99^{\text {th }}$ centile). She had had delayed eruption of her secondary dentition. She received 1:1 help for her visual impairment (with visual acuity RE 0.70 LE 0.45 corrected with +20DS both eyes) 
391 and her intellectual achievement was equivalent to her sighted peers. In addition

392 to her right 2-3 toe syndactyly, she demonstrated left $2^{\text {nd }}$ toe clinodactyly and $4^{\text {th }}$

393 toe camptodactyly. She had hypodontia, a broad bifid nasal tip, mild

394 heterochromia of the left iris, with bialteral aphakia and normal fundal

395 appearances.

396 She had normal array CGH and was diagnosed with a de novo BCOR variant

397 c.3153G>A; p.(Trp1051*) by the Deciphering Developmental Disorders (DDD)

398 study (DECIPHER ID: 262217), confirmed with Sanger sequencing (Wright,

399 Fitzgerald et al. 2015).

400

$401 \quad$ Case 11

402 Case 11 is an 11-year-old girl born by emergency Caesarian section for face

403 presentation at 42 weeks' gestation following a pregnancy complicated by

404 polyhydramnios. She has one full sister and a maternal half sister and brother, all

405 healthy. She was noted to have cleft palate, right microphthalmia, roving eye

406 movements, bilateral cataracts, ASD and patent ductus arteriosus (PDA) in the

407 neonatal period. Cataract surgery was performed at 12 and 13 weeks. She now

408 has no vision in the right eye and is partially sighted on the left. Surgery to close

409 the cleft palate was performed in infancy. The ASD and PDA closed

410 spontaneously. She had nystagmus and upslanting palpebral fissures, slit-like

411 nostrils and simple ears (Figure1aj-am). She also had hypermobility of the

412 elbows. Her first teeth erupted at one year of age and all deciduous teeth were

413 still present at the age of 7 years.

414 At the age of 11 years, she had no learning difficulties, but was assisted by a

415 teacher for the visually impaired at school. Her dentist noted fused upper right

416 central and lateral incisors and lower left lateral and central incisors. An

417 orthopantomogram performed at the age of 4 years showed at least 2 years'

418 delay of dental development and probable similar fusions in the permanent

419 dentition. No comment was made regarding root size.

420 Sequencing of $B C O R$ revealed a heterozygous nonsense variant: c.4850T $>$ G;

421 p.(Leu1617*) with complete skewing of X-inactivation. Neither parent was

422 available for genetic testing. 
425 This 3-year-old boy is the second child of non-consanguineous parents born at 42640 weeks' gestation with a birth weight of $4.040 \mathrm{~kg}$ ( $60^{\text {th }}$ centile), length $56 \mathrm{~cm}$ 427 (+1.5 SD), and head circumference $37 \mathrm{~cm}(+1 \mathrm{SD})$ (Figure 2C). The pregnancy 428 was uneventful, apart from unilateral talipes detected on scan. Echocardiography 429 shortly after birth revealed a ventricular septal defect (VSD), ASD, persistent 430 ductus arteriosus, persistent left vena cava, and non-compaction of the left 431 ventricle. Furthermore, bilateral cryptorchidism was observed. At the age of one 432 month he was admitted because of respiratory insufficiency. He also had 433 bilateral grade 4-5 vesicoureteral reflux and a single kidney stone was observed.

434 On ophthalmic assessment he had nystagmus, high myopia (-17.00 D) with 435 megalophthalmos, and posterior embryotoxon. He was noted to have full cheeks, 436 mild ptosis, exophthalmos, uplifted earlobes, a glabellar naevus flammeus, a long 437 philtrum and full nasal tip, long thumbs and left talipes (Figure 1an-ao). Brain 438 MRI showed no abnormalities. X-rays of the hand showed short metacarpals and 439 bilateral brachymesophalangy of the $5^{\text {th }}$ fingers. He developed a seizure disorder 440 from 1 year of age. His cognitive and motor milestones were severely delayed 441 and at the age of 35 months he was nonverbal, could sit, but was unable to stand. 442 His SNP array, array CGH, FISH-analysis for Pallister Killian syndrome and 443 analysis of $C H D 7, A S X L 1$ and SETBP1 were normal. WES analysis revealed a 444 hemizygous variant in BCOR (c. $4741+1 \mathrm{G}>\mathrm{A}$; p.(?)). This variant is located within 445 the donor splice site of intron 12, predicted to result in aberrant splicing Human 446 Splicing Finder tool (http://www.umd.be/HSF3/index.html) (Desmet, Hamroun 447 et al, 2009). His healthy mother is a carrier and showed 100\% skewed X448 inactivation. His healthy 4-year-old sister is also a carrier and also showed 449 skewed X-inactivation (ratio 96:4). The variant was not present in both maternal 450 grandparents and a healthy maternal uncle.

452 Case 13

453 Case 13 is a 2-year-old Caucasian girl, only child of unrelated and healthy 454 parents. There is no familiar history of congenital or developmental anomalies. 455 Pregnancy was complicated by intrauterine growth restriction in the third 456 trimester. She was born by induced delivery at 37 weeks' gestation with birth 
weight of $1.890 \mathrm{~kg}\left(<3^{\text {rd }}\right.$ centile $)$. At birth she showed bilateral microphthalmia and cataracts, but no other anomalies. At 2 months of age cataract surgery was performed and at 3 months of age she showed low vision and nystagmus. She

460 had normal psychomotor and cognitive development. She had late eruption of 461 her first teeth at 14 months of age and primary dentition was complete except 462 for the right lateral lower incisor. At 20 months of age she was referred for 463 genetic testing and targeted sequencing revealed a heterozygous de novo 464 nonsense variant in BCOR c.4402C>T; p.(Gln1468*), predicted as pathogenic.

\section{Case 14}

467 This 3-year-old girl is the second of two daughters born to non-consanguineous 468 parents. Her mother was diagnosed with bilateral cataracts at 7 months of age,

469 which had been attributed to maternal rubella infection in pregnancy. She also 470 had dental abnormalities with radiculomegaly and thin enamel. Case 14 was 471 delivered at 35 weeks due to placental failure and had breathing difficulties at 472 birth necessitating 4 days of ventilator support. She was diagnosed with bilateral 473 congenital cataracts and underwent surgery to the right eye at 7 weeks of age.

474 She also had right microphthalmia, a small restricted perimembranous VSD and 475 secundum ASD. The VSD spontaneously closed and the ASD did not require any 476 intervention. She had a thyroglossal cyst that required intravenous antibiotics 477 and drainage. Primary dentition was delayed with eruption of first teeth at 18 478 months and oligodontia (Figure 1ap-ar). Her development was normal. The DDD 479 study (Decipher ID: 303226) identified a maternally inherited heterozygous 480 frameshift variant, c.4601_4602insCT; p.(His1535CysfsTer34) in BCOR.

Case 15

483 Case 15 is a 14-year-old Caucasian girl, the third child of non-consanguineous 484 parents. There was no relevant family history. She was born following a normal 485 pregnancy and was mildly oedematous and anaemic at birth. A cleft palate was identified and she was also found to have a cardiac defect, which closed spontaneously. At nine weeks of age bilateral cataracts were diagnosed, which were surgically removed by 12 weeks of age. She then developed pupil block glaucoma in her left eye, which required surgery. She has ongoing problems with 
490 bilateral glaucoma. She also has hypermobility of hips, knees and ankles, but this

491 is improving.

492 Developmentally there have been no concerns about achieving milestones. She

493 attended a school for the visually impaired previously, but is now at mainstream

494 school with some vision support. Her facial features are in keeping with a

495 diagnosis of OFCD with macrocephaly (OFC-97th centile), bilateral ptosis,

496 hypoplastic alae nasi and broad nasal tip. Her great toes are very long and she

497 has a wide sandal gap on both her feet. There is a mild alveolar cleft (forme

498 fruste) in the midline. Sanger sequencing revealed a pathogenic frameshift

499 variant, c.3116_3117dup; p.(Asp1040Lysfs*16) in BCOR; parents declined

500 testing.

501

502 Case 16

503 Case 16 is a 2-month-old Caucasian female born following a normal pregnancy at

50438 weeks' gestation with a birth weight of $3.245 \mathrm{~kg}\left(50^{\text {th }}\right.$ centile) with normal

505 ultrasound scans. She presented with cleft palate and facial dysmorphism

506 consisting of square-shaped face with asymmetric microphthalmia, upslanting

507 palpebral fissures, large nasal tip with septate nasal cartilage and simple ears

508 (Figure 1as-av). She also had camptodactyly of the second and fourth toes, mild

509 cutaneous syndactyly of the second and third toes and long, large halluces and

510 congenital heart anomalies, consisting of a large ASD and two VSDs. In addition

511 to bilateral microphthalmia, her eye examination revealed bilateral congenital

512 cataract, iris rubeosis and flat anterior chambers. She is being investigated for

513 hearing loss, since the auditory evoked potentials were negative. As she also

514 exhibited hypotonia and abnormal movements, brain MRI was performed and

515 showed asymmetrical pontocerebellar hypoplasia, cerebral atrophy and

516 enlargement of the ventricles without obstruction. Electroencephalogram was

517 normal.

518

519 Molecular analysis of $B C O R$ revealed a de novo deletion of the exons 7 to 15,

520 confirmed by array CGH Xp11.4 (39910845_39922793)x1 (Agilent 60k ISCA)

521 and qPCR. In addition, there was a $162 \mathrm{~kb}$ deletion in 2p15 (arr[GRCh37]

522 2p15[63190016_63352116]x1) that includes OTX1 and the 3' region of WDPCP. 
523 This second CNV is of unknown significance, and could explain the neurologic

524 phenotype since OTX1 has a putative role in brain development.

526 Summary of our cases and previously published BCOR cases (Supplementary

527 Tables 1, 2, and 3)

529 Including the cases presented in this paper, a total of 95 cases from 66 families

530 harbouring pathogenic BCOR variants have been described in the literature. We

531 have summarized the findings of our cases in Table 1, and of all published cases

532 including ours in supplementary Table 1. This includes 85 heterozygous (female)

533 OFCD cases from 58 families (also detailed in Supplementary table 2) and 10

534 hemizygous (male) cases from 8 families (also detailed in Supplementary Table

$5353)$.

536

537 Discussion

538 Pathogenic variants in BCOR have been associated with two distinct phenotypes.

539 The first is the OculoFacioCardioDental (OFCD) X-linked dominant syndrome,

540 affecting exclusively females, presumed male lethal, and caused by a variety of

541 null BCOR variants. The second is a severe X-linked recessive microphthalmia

542 syndrome ('Lenz') affecting males only and caused in the majority of cases to

543 date by a specific missense variant, c.254C > T, predicting a p.(Pro85Leu)

544 substitution at the protein level. However, in this report we present additional

545 male phenotypes associated with novel BCOR variants that include

546 developmental delay in the absence of eye anomalies in 2 brothers, and one male

547 with high myopia, megalophthalmos, posterior embryotoxon, severe

548 developmental delay, and heart and bone anomalies. We also describe one male

549 with severe ocular involvement, but without psychomotor delay, harbouring the

550 previously described p.(Pro85Leu) variant.

552 We reviewed 85 OFCD cases from 58 families with pathogenic BCOR variants in

553 the literature, including the new cases described here (Supplementary Table 1).

554 Many have been recently summarized in the article by Feberwee and colleagues

555 (Feberwee, Feenstra et al. 2014). Although the classic phenotypic characteristics 
556 of OFCD (eye anomalies, craniofacial anomalies, cardiac anomalies and dental anomalies) occurred in a majority of the described cases, only $41 \%$ of cases had anomalies in all four categories. In addition to these classical characteristics, skeletal anomalies were frequently observed: $82 \%$ of cases had digit anomalies;

$56013 \%$ had radioulnar synostosis and 10\% had vertebral anomalies. Strikingly,

561 hearing loss, which has not previously been highlighted as a feature of OFCD, was

562 present in $9 \%$ of published cases. This cannot solely be attributed to secretory

563 otitis media relating to cleft palate, as only two out of the nine cases with hearing

564 loss had cleft palate. One of our cases (16) had presumed hearing loss as

565 indicated by negative auditory evoked potentials, but this case also had other 566 brain anomalies.

568 Apart from one mosaic case, all cases presented with features characteristic of 569 OFCD, which suggests complete penetrance for the protein truncating BCOR 570 variants underlying OFCD. All non-mosaic individuals, as well as three mosaic 571 cases, manifested congenital or early onset cataract, with or without additional 572 ocular features, such as microphthalmia, coloboma, lens dislocation, optic disc 573 dysplasia, secondary glaucoma and retinal detachment (the latter two possible 574 sequelae of early cataract surgery). The facial features include separated nasal 575 cartilage, high nasal bridge, long narrow face, palate/uvula anomalies, and 576 simple ears (Ng, Thakker et al. 2004, Hilton, Johnston et al. 2009, Davoody, Chen 577 et al. 2012), with features not universally described in OFCD cases (see Figure 1).

578 Cardiac anomalies, including septal defects, patent ductus arteriosus, double 579 outlet right ventricle, Fallot's tetralogy, and dextrocardia were reported in 63\% 580 of individuals. The dental anomalies can affect primary and secondary dentition 581 and can show a virtually pathognomonic radiculomegaly, or delayed, persistent 582 or unerupted primary and/or secondary dentition, hypodontia, duplication or 583 fusion of teeth (Kantaputra 2014) and are illustrated in Figure 1. Only four cases 584 were reported to be without dental anomalies. The skeletal anomalies included 585 2-3 toe syndactyly, broad halluces, hammer toes, camptodactyly, short fingers, 586 radioulnar synostosis, scoliosis, and vertebral fusion (Figure 1). 
We would like to highlight some additional features of OFCD. Mild developmental delay was present in around $10 \%$ of cases. Strikingly, hearing deficits, which are not usually described as part of the OFCD spectrum, occurred in $9 \%$ of cases, and should be considered as a new feature of this syndrome. Two

592 individuals in our series had joint hypermobility, also described once before.

593 Although this a relatively common finding in children in the general population,

594 further studies would help to determine if it is a manifestation of OFCD. Other

595 findings include: intrauterine growth retardation, poor feeding/reflux,

596 vesicoureteral reflux and asplenia, growth hormone deficiency, delayed bladder

597 control, decreased reflexes, thyroglossal cysts, lipoma in the thyroid lobe, lipoma

598 of the corpus callosum and other brain anomalies. We suggest that neuropathy

599 or muscle hypotonia, pituitary underdevelopment and lipoma may be additional

600 features of the OFCD syndrome. This paper is the first to describe a childhood

601 lymphoma in an OFCD case. This case highlights the importance of follow-up of

602 OFCD cases, and indicates that further research is needed to investigate whether 603 the occurrence of childhood or adult tumours is more common in OFCD cases 604 compared to the general population, especially in view of the tumour suppressor 605 role of BCOR described below. Interestingly haemangiomas seem to be a 606 frequent feature, and were also seen in one of our carrier females, although are 607 relatively common in the general population. Case 16 had a distinct neurological 608 phenotype that included pontocerebellar hypoplasia, cerebral atrophy and 609 enlargement of the ventricles. She had a deletion of exons 7-15 of BCOR and an 610 additional $162 \mathrm{~kb}$ deletion in 2p15 that included OTX1 and the 3' region of 611 WDPCP. The OTX1 deletion may be contributing to the neurological phenotype, 612 since mice with deletions in Otx1 have brain anomalies (Acampora, Mazan et al. 613 1996).

615 The majority of heterozygous variants in OFCD cases were predicted to cause 616 protein truncation, with $48 \%$ of them causing a frameshift, 33\% nonsense, and $6177 \%$ affecting splicing. The remaining 12\% of cases harboured a whole or partial 618 gene deletion. In $26 \%$ of OFCD cases, the condition was familial and in $74 \%$ it 619 was sporadic or unknown. For all apparently sporadic cases where parental 620 samples were analysed (35\%), the variant appeared de novo, suggesting that 
621 protein-truncating variants, including nonsense and frameshift mutations, are

622 fully penetrant. However, the possibility of gonosomal mosaicism could not be

623 excluded.

624

625 Lenz first described his microphthalmia syndrome in 1955 in an X-linked

626 pedigree manifesting male cases with severe microphthalmia syndrome with

627 delayed development, palatal and dental anomalies, skeletal anomalies,

628 congenital heart defects, unilateral renal aplasia and cryptorchidism (Lenz

629 1955). Since this paper, it is clear that many descriptions have loosely referred to

630 male patients with severe microphthalmia as having 'Lenz microphthalmia', both

631 sporadic male cases and those with an X-linked pedigree. Although due credit

632 should be attributed to Lenz for drawing attention to this severe microphthalmia

633 affecting males, the generic use of the term 'Lenz' microphthalmia to describe

634 affected males with severe syndromic microphthalmia is perhaps best avoided,

635 since it is a genetically heterogeneous group (Traboulsi, Lenz et al. 1988, Hilton,

636 Johnston et al. 2009). Hilton and colleagues analysed 21 male patients with

637 presumed 'Lenz' microphthalmia and identified 1 with the typical c.254C>T;

638 p.(Pro85Leu) missense variant in $B C O R$, demonstrating that $B C O R$ is not the

639 major cause of severe male microphthalmia, a finding supported by other groups

640 (Ng et al. 2004)(Horn, Chyrek et al. 2005)(Hilton et al. 2009, Suzumori et al.

641 2013).

642

643 The phenotypes of the hemizygous male cases with BCOR variants partially

644 overlaps with the female cases, with eye, craniofacial, cardiac and skeletal

645 anomalies present in the majority of male cases. Dental anomalies were not

646 reported in this group, whereas half of these cases presented with

647 developmental delay, and 40\% with genitourinary anomalies. No protein

648 truncating variants have been described in male cases, with missense variants in

6497 families and a splice site variant in another one.

650

651 Our case 2 with the typical c.254C>T; p.(Pro85Leu) shows an interesting

652 phenotype, displaying additional features to previous descriptions. He shows a

653 severe microphthalmia phenotype, with developmental delay associated with 
654 autistic features, short stature, cardiac anomalies, broad halluces, long thin

655 fingers, vesico-ureteric reflux, cryptorchidism, hypotonia, reduced muscle mass,

656 scoliosis, and large low set ears in the absence of microcephaly. However, case 9,

657 who displays high intelligence and no autistic features and also carrying

658 c.254C>T; p.(Pro85Leu), clearly demonstrates that males with the typical BCOR

659 variant do not universally display these features. The phenotype of severe eye

660 anomalies plus cryptorchidism, hypotonia, and autistic features in the male

661 BCOR-related syndrome shows some overlap with SOX2 anophthalmia syndrome

662 (Fantes, Ragge et al. 2003, Ragge, Lorenz et al. 2005, Bakrania, Robinson et al.

663 2007), such that male cases with severe microphthalmia or anophthalmia,

664 developmental delay, reduced growth and cryptorchidism, might benefit from

665 panel testing that includes both SOX2 and BCOR, Specific features, such as lack of

666 developmental delay with presence of other extraocular features including long

667 thin fingers or toes, large ears, cardiac anomalies, vesicoureteric reflux in

668 association with severe bilateral eye anomalies might suggest BCOR is more

669 likely to be the responsible gene.

670

671 Recently Zhu and colleagues described a boy with multiple birth anomalies,

672 congenital glaucoma, AV canal type ventricular septal defect and cerebral white

673 matter hypoplasia (Zhu, Dai et al. 2015). Molecular testing revealed a de novo

674 novel missense variant in BCOR (c.1619G>A; p.[Arg540Glu]) predicted to be

675 'probably damaging'. As the authors indicated, it was unclear if the variant in

$676 B C O R$, although suggestive, was the underlying diagnosis. However, in view of

677 posterior embryotoxon seen in our cases, which can be part of anterior segment

678 dysgenesis, and can be associated with primary glaucoma, this might imply that

679 posterior embryotoxon is part of the spectrum of eye anomalies associated with

$680 B C O R$ variants. Furthermore, our case 12 (see below) with posterior

681 embryotoxon with megalophthalmos and myopia had a novel splice site variant

682 in $B C O R$ (c. $4741+1 \mathrm{G}>\mathrm{A}$; p.[?]).

683

684 Cases 4 and 5 are two half-brothers who have an interesting constellation of

685 features that include early neonatal diabetes, hypotonia, ASD, bilateral posterior

686 embryotoxon (without cataract), long slender fingers, camptodactyly, 

haemangiomas, cleft palate, posterior arachnoid cyst and severe growth and intellectual delay (in the older boy). The younger, but not the older brother, received WES and this revealed the c.4807A>C; p.(Ser1603Arg) BCOR variant present in his affected brother and his unaffected mother, but absent in his unaffected half-brothers. There are enough features of $B C O R \mathrm{X}$-linked syndrome to suggest this as the underlying diagnosis. However, as this is the first description of an intellectual disability syndrome associated with $B C O R$, without the characteristic findings of microphthalmia, this gene should be considered in other males with intellectual disability with or without overlapping features to explore this potential new phenotype more fully.

Case 12 showed a boy with high myopia and large globes and who also demonstrates a splice site variant, and therefore distinct from the classical missense variant c.254C>T; p.(Pro85Leu), seen in males with severe microphthalmia. The organs involved in the phenotype in this boy overlap with OFCD syndrome and/or Lenz microphthalmia. However, his eye phenotype is distinct from the phenotype of those two disorders in causing increased ocular growth and myopia, and he additionally showed posterior embryotoxon, also seen in cases 4 and 5. Interestingly, his unaffected mother and sister who carry the same variant show highly skewed X-inactivation.

The mechanism by which $B C O R$ acts to promote eye growth is not precisely known. Loss of bcor function leads to coloboma formation in the zebrafish. Through evidence from oncogenic pathways, it is known that the BCOR/BCL6/SIRT1 complex interacts with the SHH signaling pathway, also important in human eye development (and medulloblastoma) (Tiberi, Bonnefont et al. 2014). In zebrafish, the bcor/bcl6a complex appears to interact with hdacs, and there is some evidence that part of the mechanism may occur by bcor/bcl6a and Hdac1 co-repressing p53 expression, although there is no evidence that humans with germline p53 mutations have developmental eye anomalies (Lee, Lee et al. 2013). 
BCOR is a co-repressor that interacts with BCL6 at the POZ domain. BCL6 is an oncogene important in B cell development and oncogenesis. It encodes a zincfinger transcriptional repressor, which is a regulator of germinal centre formation. BCOR aberrations have been identified in extranodal NK/T cell lymphomas and in secondary acute myeloid leukemias, and other tumours (Dobashi, Tsuyama et al. 2016). Furthermore, Tanaka and colleagues (Tanaka, Nakajima-Takagi et al. 2017) have demonstrated a likely tumour suppressor role for BCOR in T lymphocytes in mice. This provides supporting evidence that $\mathrm{T}$ cell lymphoma described in case 3 may be linked to the germline BCOR variant in this patient. The role of $B C O R$ in tumorigenesis does not appear to be limited to tumour suppression. Various BCOR rearrangements, including in frame internal tandem duplications of $B C O R$ exon 15 and gene fusions involving BCOR, illustrate an emerging role in tumour enhancement in various sarcoma subtypes (Pierron, Tirode et al. 2012, Ueno-Yokohata, Okita et al. 2015).

There appears to be interesting genotype-phenotype correlation in BCOR-related conditions. Affected males tend to have hypomorphic missense variants, although some interesting new phenotypes are emerging with missense and splice variants, and their carrier mothers are unaffected (although they have skewed X-inactivation). In contrast, affected females with OFCD have protein truncating variants or partial/whole gene deletions, and exhibit skewed Xinactivation. If the OFCD is inherited from their mothers, the mothers will also express the disorder, show skewed X-inactivation, and the variant is presumed lethal in affected male offspring as evidenced by miscarriages.

This paper has aimed to summarize the X-linked BCOR syndrome, and to extend the phenotypic spectrum associated with $B C O R$ pathogenic variants. Females tend to have features of OFCD, but in addition can manifest further features, including neuropathy, muscle hypotonia, pituitary underdevelopment, lipoma and lymphoma. We have shown that males with the typical BCOR variant c.254C>T; p.(Pro85Leu), contrary to existing information, can have normal intellectual development. We have also demonstrated that new variants in $B C O R$ can be associated with X-linked syndromic intellectual disability in males, and 
752 megalophthalmos and myopia, thus extending the phenotype. We would

753 recommend that males with severe microphthalmia or anophthalmia and

754 relevant extraocular features described be tested for SOX2 and BCOR as part of a

755 panel. Furthermore, females with early onset cataract should be examined for

756 extraocular features of the OFCD syndrome, and if any present tested for BCOR

757 variants, with the caveat that occasionally an ocular-only phenotype can exist. In

758 view of our cases with posterior embryotoxon or megalophthalmos, we suggest

759 that individuals with similar phenotypes that include suggestive extraocular

760 features are tested for BCOR variants. Furthermore, we would recommend long

761 term multicentre follow-up studies of individuals with BCOR pathogenic variants

762 to determine the incidence of tumour formation. We would also propose

763 abandoning the use of the generic term 'Lenz' microphthalmia since this refers to

764 Lenz' clinical description of a particular pedigree with a severe microphthalmia

765 phenotype affecting males and is not representative of a genetically defined

766 syndrome. Instead, we suggest a new term referring to BCOR conditions as X-

767 linked BCOR-related syndrome, specifying male or female as appropriate. 
Legends:

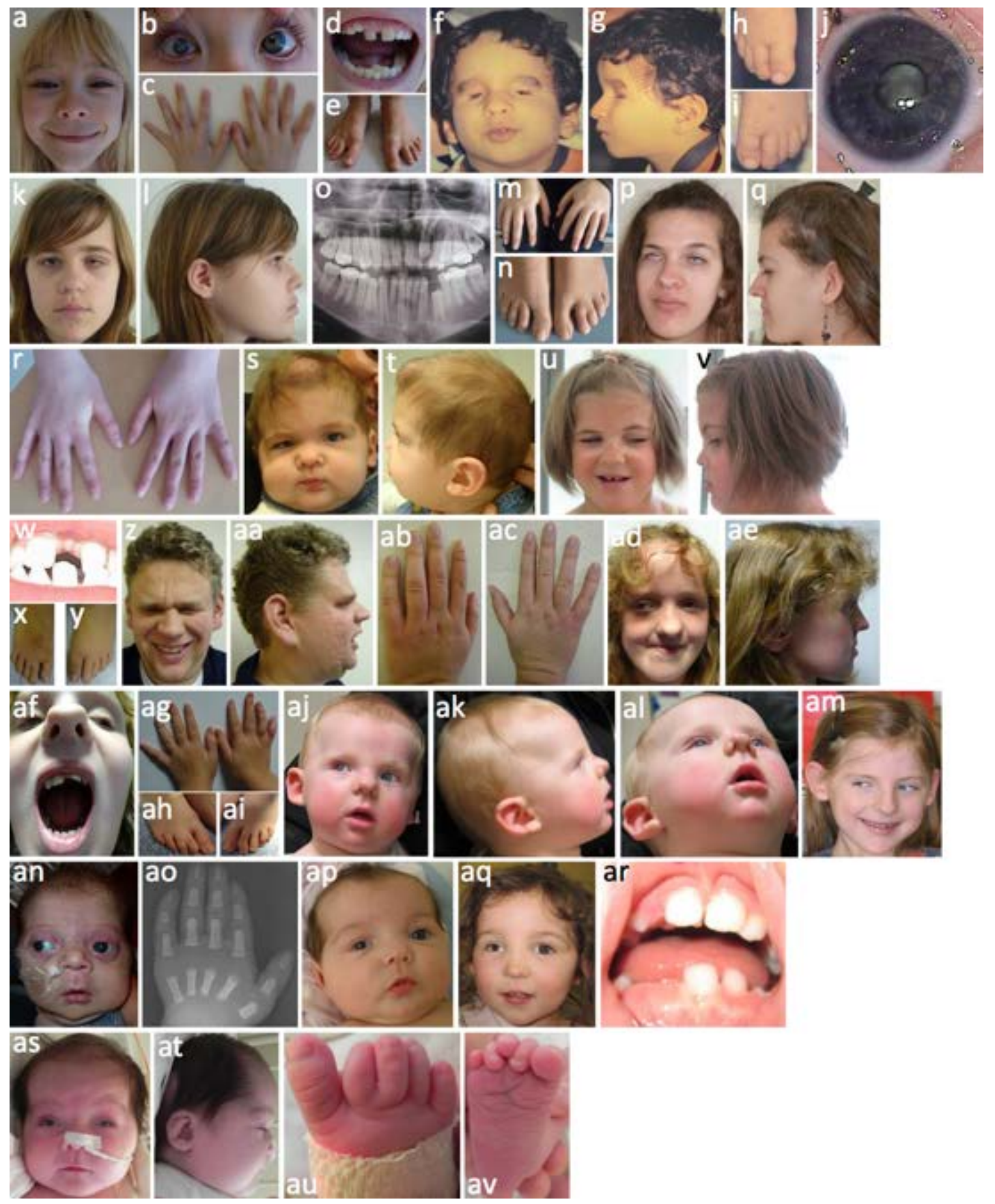

Figure 1 - Clinical Photographs

a-e - case 1 showing broad nasal base, right microphthalmia, tooth

abnormalities, long slender fingers, increased sandal gap, and right second toe clinodactyly

f-i - case 2 showing bilateral severe microphthalmia, downslanting palpebral fissures, thick eyebrows, a broad nasal root and tip, a long philtrum, large low set posteriorly rotated ears, and broad halluces 
j - case 3 - eye photograph demonstrating congenital cataract

$\mathrm{k}-\mathrm{o}$ - case 6 showing short bulbous nose, small ears and prognathism, $5^{\text {th }}$ finger

clinodactyly and 2-3 partial syndactyly of the left toes and orthopantomogram showing radiculopathy

p-r - case 7 showing bilateral microphthalmia, broad nose, and long, slender

fingers

$\mathrm{s}-\mathrm{w}$ - case 8 showing large hemangioma on the forehead, and tooth anomalies

with agenesis of both superior lateral incisors

$\mathrm{x}$-ac - case 9 showing bilateral anophthalmia, relatively large ears, partial 2-3 toe

syndactyly

ad-ai - case 10 showing bilateral microphthalmia, prominent forehead, flat nasal

bridge, upturned nose with a broad bifid tip, hypodontia, mesaxial

polysyndactyly of the $4^{\text {th }}$ digit with $5 / 6$ syndactyly of the right hand partial $2 / 3$

syndactyly of the right toes

aj-am - case 11 showing right microphthalmia, upslanting palpebral fissures,

broad nasal tip with slit-like nostrils and simple ears

an-ao - case 12 showing bilateral megalophthalmos and exophthalmos, full

cheeks, uplifted earlobes, long philtrum and full nasal tip, long thumbs and left

talipes; (ao) short metacarpals and brachymesophalangy $5^{\text {th }}$ fingers

ap-ar - case 14 showing right microphthalmia and oligodontia

as-av - case 16 showing asymmetric microphthalmia, upslanting palpebral

fissures, large nasal tip (obscured by tape), simple ears; (au-av) showing

camptodactyly of second and fourth toes, mild cutaneous syndactyly of second and third toes and long, large halluces. 
A
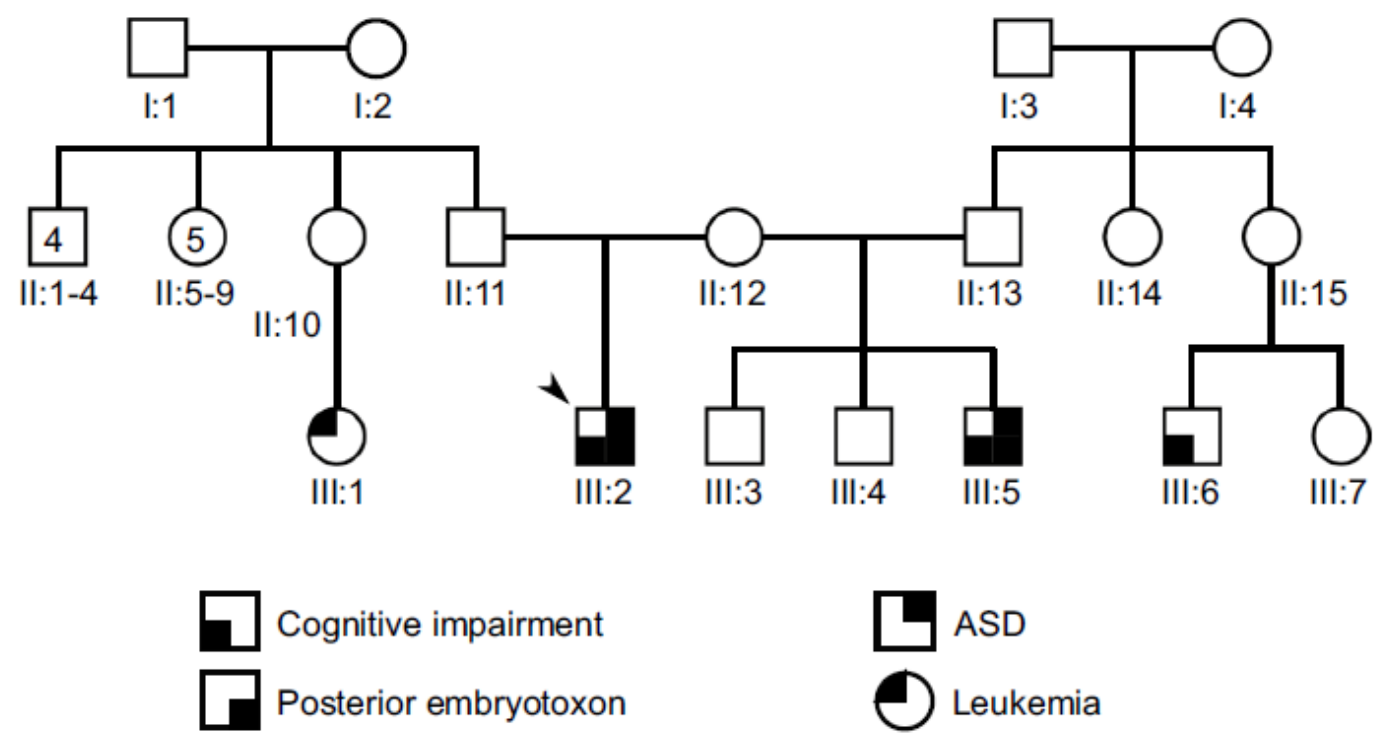

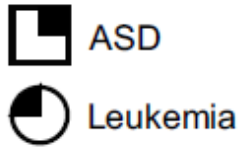

B

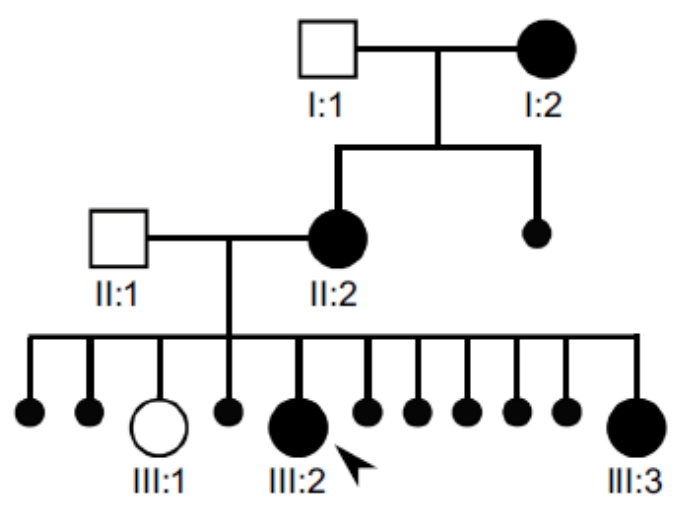

C

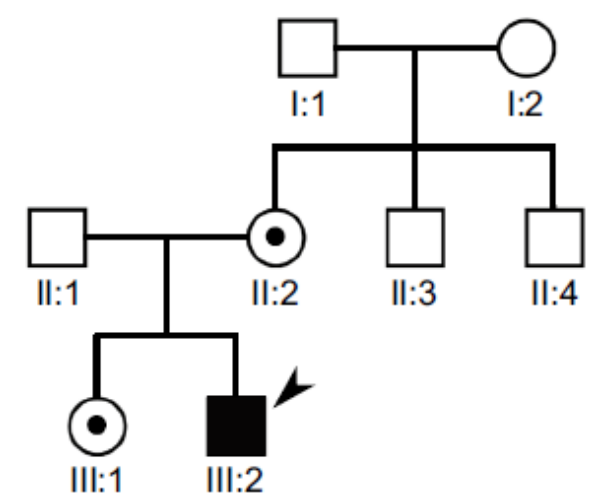

Figure 2. A. Pedigree of cases 4 and 5; B. Pedigree of case 7; C. Pedigree of case 12 


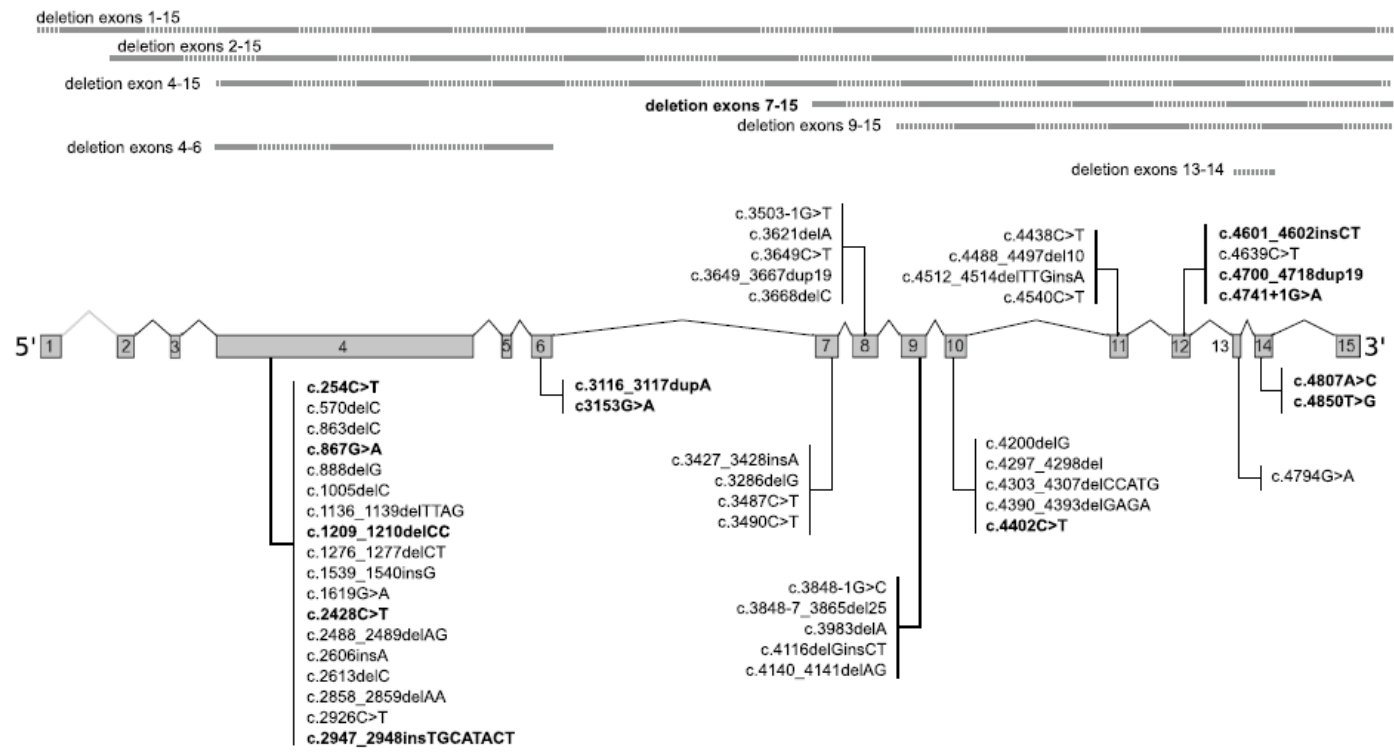

Figure 3: Summary of the described and new (in bold) variants in $B C O R$

Tables:

Table 1: Summary of Phenotypic Findings

Supplementary Table 1: Summary of Clinical Features and Variants of Published cases identified with BCOR variants (including current series)

Supplementary Table 2: Summary of OFCD cases

Supplementary Table 3: Summary of X-linked BCOR male cases 


\section{Table 1: Summary of Phenotypic Findings}

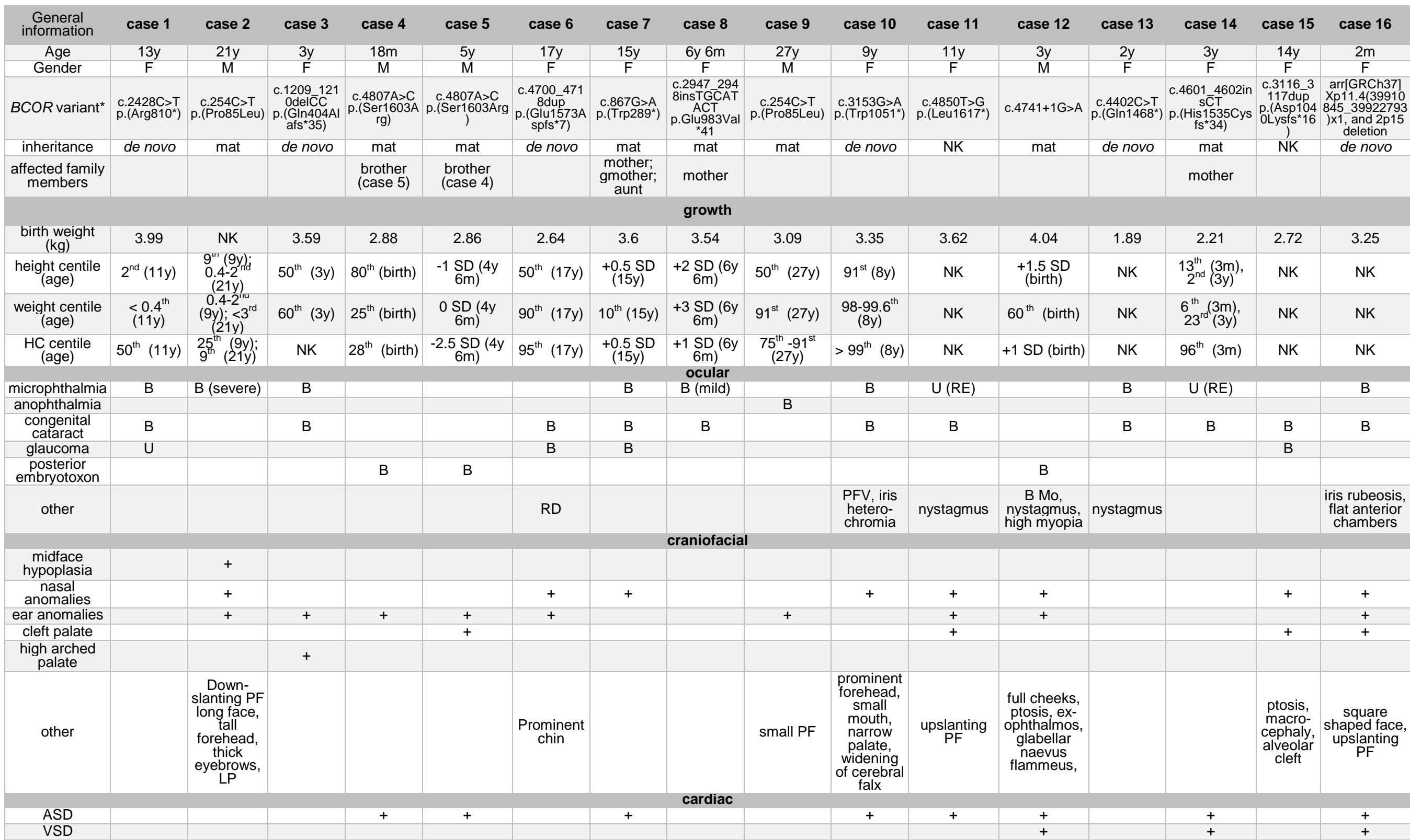




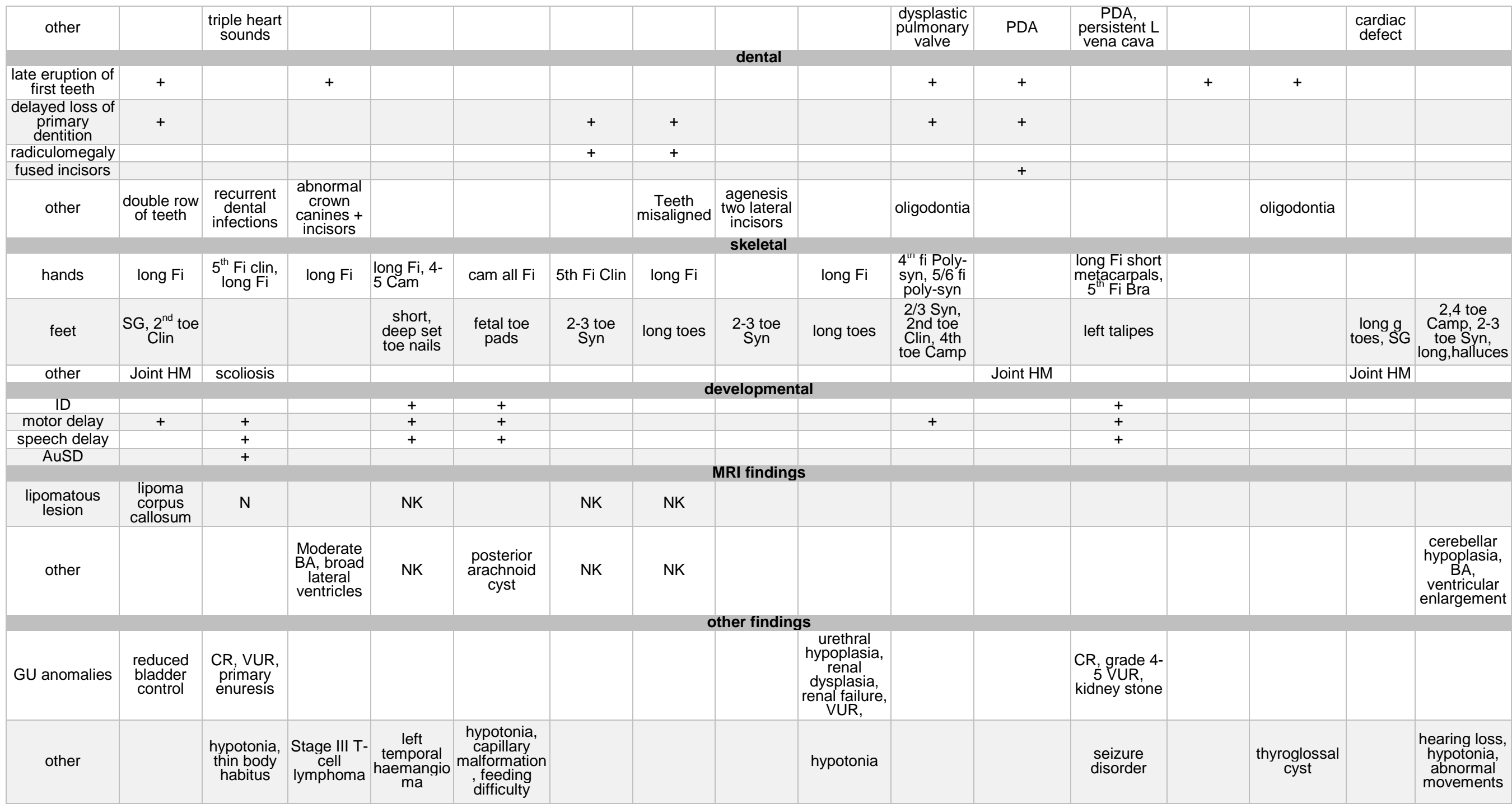

\section{Abbreviations:}

* (NM_001123385.1)

ASD, atrial septal defect; AuSD, autistic spectrum disorder; B, bilateral; BA, brain atrophy; Bra, brachymesophalangy ; Cam, camptodactyly; Clin, clinodactyly; CR, cryptorchidism; F, female; Fi, fingers; gmother, grandmother; GU, genitourinary; ID, Intellectual delay, Joint HM, joint hypermobility; kg, kilogram; LE, left eye; LP, long philtrum; M, male; m, months; mat, maternal; Mo,

Megalophthalmos; MRI, magnetic resonance imaging; N, normal; NK, not known; pat, paternal; PDA, patent ductus arteriosus; PF, palpebral fissures; PFV, persistent fetal vasculature; RD, retinal detachment; RE, right eye; SD, standard deviation; SG, increased sandal gap; Syn, syndactyly; U, unilateral; VSD, ventriculoseptal defect; VUR, vesicoureteric reflux; y, year 
Conflict of Interest Statement

On behalf of all authors, the corresponding author states that there is no conflict of interest. 
References

Acampora, D., Mazan, S., Avantaggiato, V., Barone, P., Tuorto, F., Lallemand, Y., Brulet, P. \& Simeone, A. (1996) Epilepsy and brain abnormalities in mice lacking the Otx1 gene. Nature Genet., 14, 218-222.

Adzhubei IA, Schmidt S, Peshkin L, Ramensky VE, Gerasimova A, Bork P, et al. (2010) A method and server for predicting damaging missense mutations. Nat Methods 7: 248-249.

Amos-Landgraf, J M., Cottle A, Plenge R M., Friez M, Schwartz C E., Longshore J, and Willard H F. (2006) X Chromosome-Inactivation Patterns of 1,005 Phenotypically Unaffected Females. Am. J. of Hum. Genet:79:493-499 Bakrania, P., D. O. Robinson, D. J. Bunyan, A. Salt, A. Martin, J. A. Crolla, A. Wyatt, A. Fielder, J. Ainsworth, A. Moore, S. Read, J. Uddin, D. Laws, D. Pascuel-Salcedo, C. Ayuso, L. Allen, J. R. Collin and N. K. Ragge (2007). "SOX2 anophthalmia syndrome: 12 new cases demonstrating broader phenotype and high frequency of large gene deletions." Br J Ophthalmol 91(11): 1471-1476.

Danda S, van Rahden VA, John D, Paul P, Raju R, Koshy S, Kutsche K (2014). "Evidence of Germline Mosaicism for a novel BCOr mutation in two indian sisters with oculo-facio-cardio dental Syndrome". Mol Syndromol. 5(5):251-6 Davoody, A., I. P. Chen, R. Nanda, F. Uribe and E. J. Reichenberger (2012). "Oculofaciocardiodental syndrome: a rare case and review of the literature." Cleft Palate Craniofac I 49(5): e55-60.

Desmet FO, Hamroun D, Lalande M, Collod-Beroud G, Claustres M, Beroud C. Human Splicing Finder: an online bioinformatics tool to predict splicing signals. Nucleic Acids Res. 2009 May;37(9):e67

Di Stefano, C., B. Lombardo, C. Fabbricatore, C. Munno, I. Caliendo, F. Gallo and L. Pastore (2015). "Oculo-facio-cardio-dental (OFCD) syndrome: the first Italian case of BCOR and co-occurring OTC gene deletion." Gene 559(2): 203-206. Dobashi, A., N. Tsuyama, R. Asaka, Y. Togashi, K. Ueda, S. Sakata, S. Baba, K. Sakamoto, K. Hatake and K. Takeuchi (2016). "Frequent BCOR aberrations in extranodal NK/T-Cell lymphoma, nasal type." Genes Chromosomes Cancer 55(5): 460-471. 
Esmailpour, T., H. Riazifar, L. Liu, S. Donkervoort, V. H. Huang, S. Madaan, B. M. Shoucri, A. Busch, J. Wu, A. Towbin, R. B. Chadwick, A. Sequeira, M. P. Vawter, G. Sun, J. J. Johnston, L. G. Biesecker, R. Kawaguchi, H. Sun, V. Kimonis and T. Huang (2014). "A splice donor mutation in NAA10 results in the dysregulation of the retinoic acid signalling pathway and causes Lenz microphthalmia syndrome." I Med Genet 51(3): 185-196.

Fantes, J., N. K. Ragge, S. A. Lynch, N. I. McGill, J. R. Collin, P. N. Howard-Peebles, C. Hayward, A. J. Vivian, K. Williamson, V. van Heyningen and D. R. FitzPatrick (2003). "Mutations in SOX2 cause anophthalmia." Nat Genet 33(4): 461-463. Feberwee, H. E., I. Feenstra, S. Oberoi, I. E. Sama, C. W. Ockeloen, F. Clum, A. Slavotinek, M. A. Kuijpers, D. Dooijes, A. M. Kuijpers-Jagtman, T. Kleefstra and C. E. Carels (2014). "Novel BCOR mutations in patients with oculofaciocardiodental (OFCD) syndrome." Clin Genet 85(2): 194-197.

Fujimaki, T., Yokoyama T., Kawano, H., Fujiki, K., Ikeda, M., Murakami, A. (2008).

"A Case Report of Oculofaciocardiodental Syndrome with novel BCOR gene mutation." Investigative Ophthalmology \& Visual Science 49 (13): 465 Hilton, E., J. Johnston, S. Whalen, N. Okamoto, Y. Hatsukawa, J. Nishio, H. Kohara, Y. Hirano, S. Mizuno, C. Torii, K. Kosaki, S. Manouvrier, O. Boute, R. Perveen, C. Law, A. Moore, D. Fitzpatrick, J. Lemke, F. Fellmann, F. G. Debray, F. Dastot-LeMoal, M. Gerard, J. Martin, P. Bitoun, M. Goossens, A. Verloes, A. Schinzel, D. Bartholdi, T. Bardakjian, B. Hay, K. Jenny, K. Johnston, M. Lyons, J. W. Belmont, L. G. Biesecker, I. Giurgea and G. Black (2009). "BCOR analysis in patients with OFCD and Lenz microphthalmia syndromes, mental retardation with ocular anomalies, and cardiac laterality defects." Eur I Hum Genet 17(10): 1325-1335. Horn, D., M. Chyrek, S. Kleier, S. Luttgen, H. Bolz, G. K. Hinkel, G. C. Korenke, A. Riess, C. Schell-Apacik, S. Tinschert, D. Wieczorek, G. Gillessen-Kaesbach and K. Kutsche (2005). "Novel mutations in BCOR in three patients with oculo-faciocardio-dental syndrome, but none in Lenz microphthalmia syndrome." Eur J Hum Genet 13(5): 563-569.

Jiang YH, Fang P, Adesina AM, Furman P, Johnston JJ, Biesecker LG, Brown CW. (2009). "Molecular characterization of co-occurring Duchenne muscular dystrophy and X-linked oculo-facio-cardio-dental syndrome in a girl" Am J Med Genet A. 149A(6):1249-52 
Kantaputra, P. N. (2014). "BCOR mutations and unstoppable root growth: a commentary on oculofaciocardiodental syndrome: novel BCOR mutations and expression in dental cells." L Hum Genet 59(6): 297-299.

Kondo, Y., H. Saitsu, T. Miyamoto, K. Nishiyama, Y. Tsurusaki, H. Doi, N. Miyake, N. K. Ryoo, J. H. Kim, Y. S. Yu and N. Matsumoto (2012). "A family of oculofaciocardiodental syndrome (OFCD) with a novel BCOR mutation and genomic rearrangements involving NHS." ЦHum Genet 57(3): 197-201.

Lee, J., B. K. Lee and J. M. Gross (2013). "Bcl6a function is required during optic cup formation to prevent p53-dependent apoptosis and colobomata." $\mathrm{Hum} \mathrm{Mol}$ Genet 22(17): 3568-3582.

Lek, M, K J. Karczewski, E. V. Minikel, K E. Samocha, E Banks, T Fennell, et al. ((2016),. "Analysis of protein-coding genetic variation in 60,706 humans", Nature 536: 285-291 Lenz, W. (1955). "[Recessive, sex-limited microphthalmia with multiple abnormalities]." Z Kinderheilkd 77(4): 384-390.

Lozic, B., J. Ljubkovic, D. G. Panduric, I. Saltvig, K. Kutsche, V. Krzelj and T. Zemunik (2012). "Oculo-facio-cardio-dental syndrome in three succeeding generations: genotypic data and phenotypic features." Braz J Med Biol Res 45(12): 1315-1319.

Ma, A. S., J. R. Grigg, G. Ho, I. Prokudin, E. Farnsworth, K. Holman, A. Cheng, F. A. Billson, F. Martin, C. Fraser, D. Mowat, J. Smith, J. Christodoulou, M. Flaherty, B. Bennetts and R. V. Jamieson (2016). "Sporadic and Familial Congenital Cataracts: Mutational Spectrum and New Diagnoses Using Next-Generation Sequencing." Hum Mutat 37(4): 371-384.

Ng, D., N. Thakker, C. M. Corcoran, D. Donnai, R. Perveen, A. Schneider, D. W. Hadley, C. Tifft, L. Zhang, A. O. Wilkie, J. J. van der Smagt, R. J. Gorlin, S. M. Burgess, V. J. Bardwell, G. C. Black and L. G. Biesecker (2004). "Oculofaciocardiodental and Lenz microphthalmia syndromes result from distinct classes of mutations in BCOR." Nat Genet 36(4): 411-416.

Ng PC, Henikoff S. SIFT: predicting amino acid changes that affect protein function. Nucleic Acids Res 2003; 31: 3812-3814. 
O’Byrne JJ, Laffan E, Murray DJ, Reardon W (2017). “Oculo-facio-cardio-dental syndrome with craniosynostosis temporal hypertrichosis, and deafness." Am J Med Genet A. 173(5):1374-1377

Oberoi, S., A. E. Winder, J. Johnston, K. Vargervik and A. M. Slavotinek (2005). "Case reports of oculofaciocardiodental syndrome with unusual dental findings." Am J Med Genet A 136(3): 275-277.

Pierron, G., F. Tirode, C. Lucchesi, S. Reynaud, S. Ballet, S. Cohen-Gogo, V. Perrin, J. M. Coindre and O. Delattre (2012). "A new subtype of bone sarcoma defined by BCOR-CCNB3 gene fusion." Nat Genet 44(4): 461-466.

Ragge, N. K., B. Lorenz, A. Schneider, K. Bushby, L. de Sanctis, U. de Sanctis, A. Salt, J. R. Collin, A. J. Vivian, S. L. Free, P. Thompson, K. A. Williamson, S. M. Sisodiya, V. van Heyningen and D. R. Fitzpatrick (2005). "SOX2 anophthalmia syndrome." $\underline{\mathrm{Am}}$ LMed Genet A 135(1): 1-7; discussion 8.

Scott, A. F., D. W. Mohr, L. M. Kasch, J. A. Barton, R. Pittiglio, R. Ingersoll, B. Craig, B. A. Marosy, K. F. Doheny, W. C. Bromley, T. H. Roderick, N. Chassaing, P. Calvas, S. S. Prabhu and E. W. Jabs (2014). "Identification of an HMGB3 frameshift mutation in a family with an X-linked colobomatous microphthalmia syndrome using whole-genome and X-exome sequencing." ذAMA Ophthalmol 132(10): 1215-1220.

Surapornsawasd, T., T. Ogawa and K. Moriyama (2015). "Identification of nuclear localization signals within the human BCOR protein." FEBS Lett 589(21): 33133320.

Suzumori, N., T. Kaname, Y. Muramatsu, K. Yanagi, K. Kumagai, S. Mizuno, K. Naritomi, S. Saitoh and M. Sugiura-Ogasawara (2013). "Prenatal diagnosis of Xlinked recessive Lenz microphthalmia syndrome." L Obstet Gynaecol Res 39(11): 1545-1547.

Tanaka, T., Y. Nakajima-Takagi, K. Aoyama, S. Tara, M. Oshima, A. Saraya, S. Koide, S. Si, I. Manabe, M. Sanada, M. Nakayama, M. Masuko, H. Sone, H. Koseki and A. Iwama (2017). "Internal deletion of BCOR reveals a tumor suppressor function for BCOR in T lymphocyte malignancies." L Exp Med 214(10): 29012913.

Tiberi, L., J. Bonnefont, J. van den Ameele, S. D. Le Bon, A. Herpoel, A. Bilheu, B. W. Baron and P. Vanderhaeghen (2014). "A BCL6/BCOR/SIRT1 complex triggers 
neurogenesis and suppresses medulloblastoma by repressing Sonic Hedgehog signaling." Cancer Cell 26(6): 797-812.

Traboulsi, E. I., W. Lenz, M. Gonzales-Ramos, J. Siegel, W. G. Macrae and I. H. Maumenee (1988). "The Lenz microphthalmia syndrome." Am J Ophthalmol 105(1): 40-45.

Ueno-Yokohata, H., H. Okita, K. Nakasato, S. Akimoto, J. Hata, T. Koshinaga, M. Fukuzawa and N. Kiyokawa (2015). "Consistent in-frame internal tandem duplications of BCOR characterize clear cell sarcoma of the kidney." Nat Genet 47(8): 861-863.

Wildeman, M., E. van Ophuizen, J. T. den Dunnen and P. E. Taschner (2008). "Improving sequence variant descriptions in mutation databases and literature using the Mutalyzer sequence variation nomenclature checker." 29(1): 6-13.

Wright, C. F., T. W. Fitzgerald, W. D. Jones, S. Clayton, J. F. McRae, M. van Kogelenberg, D. A. King, K. Ambridge, D. M. Barrett, T. Bayzetinova, A. P. Bevan, E. Bragin, E. A. Chatzimichali, S. Gribble, P. Jones, N. Krishnappa, L. E. Mason, R. Miller, K. I. Morley, V. Parthiban, E. Prigmore, D. Rajan, A. Sifrim, G. J. Swaminathan, A. R. Tivey, A. Middleton, M. Parker, N. P. Carter, J. C. Barrett, M. E. Hurles, D. R. FitzPatrick, H. V. Firth and D. D. D. study (2015). "Genetic diagnosis of developmental disorders in the DDD study: a scalable analysis of genome-wide research data." Lancet 385(9975): 1305-1314.

Zhou, Y., A. Wojcik, V. R. Sanders, B. Rahmani and S. P. Kurup (2017). "Ocular findings in a patient with oculofaciocardiodental (OFCD) syndrome and a novel BCOR pathogenic variant." Int Ophthalmol. (epub ahead of print) Zhu, X., F. R. Dai, J. Wang, Y. Zhang, Z. P. Tan and Y. Zhang (2015). "Novel BCOR mutation in a boy with Lenz microphthalmia/oculo-facio-cardio-dental (OFCD) syndrome." Gene 571(1): 142-144. 